\title{
Effet des conditions de transport et de la conservation sur le comptage cellulaire et pertes de précision associées aux âges des échantillons de lait de vache
}

\author{
M.H. Othmane ${ }^{1 *}$ L. Trabelsi $^{2}$ \\ M. Ben Hammouda ${ }^{3}$ R. Bergaoui ${ }^{2}$
}

Mots-clés

Bovin - Lait de vache - Numération cellulaire - Réfrigération -

Préservation - Tunisie.

\begin{abstract}
Résumé
L'effet de l'agent conservateur (sans conservation, dichromate de potassium ou bronopol) et des conditions de transport (température ambiante et réfrigération) sur le taux cellulaire (SCC) de laits individuels de vaches a été étudié à deux reprises durant la lactation. Mille cinquante (expérience 1) et 924 (expérience 2) mesures de SCC ont été effectuées à l'aide d'un appareil Fossomatic à partir de laits individuels respectivement de 25 et de 22 vaches du même lot. Par ailleurs, chaque lait individuel a été divisé en plusieurs aliquotes et analysé à différents temps après le prélèvement ( 5 h, et $1,2,3,4,5$ et 6 jours) pour estimer les pertes de précision associées au temps d'analyse, selon que les aliquotes sont conservées, réfrigérées ou non. Ni l'agent conservateur ni les conditions de transport n' ont eu d'effets significatifs sur le taux cellulaire pour les deux expériences qui ont différé par le niveau cellulaire dans le lait. La précision des mesures associée à chaque âge dépendait du stade de lactation, les erreurs étant de loin faibles lorsque la quantité de lait était plus abondante et le SCC était donc moins élevé. Pour des raisons pratiques, I'analyse du lait pour le SCC devrait être effectué au plus tard dans les $48 \mathrm{~h}$ après prélèvement.
\end{abstract}

\section{INTRODUCTION}

La mammite est la maladie la plus fréquente et la plus coûteuse pour l'industrie laitière bovine (3). Ces coûts sont attribuables tant au traitement des animaux atteints qu'aux pertes causées par la diminution de la production. Les données publiées montrent qu'au fur et à mesure que le taux de cellules somatiques (SCC) augmente, la quantité de lait produite diminue et sa qualité se détériore. Il est fréquent que l'épisode de mammite ne puisse être détecté visuellement. On parle alors d'une mammite subclinique et on a recours au SCC pour tenter d'identifier les vaches atteintes.

\footnotetext{
1. Institut national de la recherche agronomique de Tunisie, Béja, Tunisie.

2. Institut national agronomique de Tunisie, Tunis, Tunisie.

3. Institution de la recherche et de l'enseignement supérieur agricoles, Tunis, Tunisie.

* Auteur pour la correspondance

Institut national de la recherche agronomique de Tunisie, UEA Lafareg, BP 260, Béja, Tunisie.

Tél. : +21678456687; fax : +21671231592

E-mail : othmane.mh@iresa.agrinet.tn
}

L'intérêt du SCC comme méthode précoce de diagnostic des mammites subcliniques est évident si l'on tient compte de la relation entre cette variable biologique et les pertes en production et en composition du lait $(6,12)$.

Il a été montré que l'infection intra mammaire par des bactéries est le facteur primordial causant l'augmentation des cellules somatiques du lait ; 90 p. 100 des augmentations du compte cellulaire y sont associées (10). Le contrôle de la mammite doit aussi passer par une bonne compréhension des facteurs qui la favorisent et des microorganismes qui la provoquent. Tous ces facteurs agissent évidemment avant l'extraction du lait qui aurait alors un taux cellulaire initial non nul. D'autres facteurs peuvent intervenir après l'extraction du lait de la mamelle et apporter des modifications sur le taux cellulaire avant d'être estimé d'une façon routinière au sein du contrôle laitier officiel.

Ce travail a eu pour objectifs : (i) l'étude de l'effet de la conservation du lait et de sa réfrigération durant le transport sur le SCC, et (ii) l'estimation des pertes de précision associées au temps écoulé entre le prélèvement et l'analyse des échantillons, par rapport à la numération cellulaire initiale prise comme référence, 
pour déterminer les délais seuils durant lesquels le SCC pourrait être analysé sans risque de déviations importantes. La mesure de référence correspond à celle du lait non réfrigéré, non conservé et analysé dès son arrivée au laboratoire.

\section{MATERIEL ET METHODES}

\section{Origine des données}

L'étude a porté sur un lot homogène de vingt-cinq vaches laitières de race Holstein relevant de la Société des fermes laitières (SFL), située dans la région de Medjez El-bab, Béja, au nord-ouest du pays. Cet élevage a eu pour objectif l'amélioration de la production laitière sur le plan quantitatif et qualitatif. Le niveau de production moyen était de 7500 1/vache/lactation pour une durée de lactation de 305 jours, soit une production journalière de $25 \mathrm{l}$. Le troupeau était inscrit au contrôle laitier officiel du type A4. Les vaches étaient traites trois fois par jour. Les échantillons de lait individuel ont tous été collectés durant la traite du matin à 6 h 00 .

Les vaches choisies répondaient aux conditions fixées par les auteurs : homogénéité pour l'âge (2 à 4 ans), stade de lactation (expérience 1 : début de lactation; expérience 2 : pleine lactation) et état sanitaire de la mamelle. Le choix de l'élevage a aussi tenu compte :

- de la localisation de la ferme par rapport au laboratoire d'analyse, à $50 \mathrm{~km}$ de distance, permettant d'étudier l'effet des conditions d'échantillonnage et de transport du lait (température, conservation, distance...) ;

- de l'effectif bovin laitier présent dans cette exploitation, assurant la constitution d'un lot de vaches homogènes selon les conditions préalablement fixées.

\section{Matériel d'échantillonnage}

Le matériel utilisé pour le prélèvement et le conditionnement des échantillons de lait a été le suivant : louches de prélèvement en acier inoxydable, récipients avec couvercles en plastique, glacières avec accumulateurs de froid, flacons de $50 \mathrm{ml}$ en plastique transparent et sans conservateur, flacons contenant les doses indiquées de l'agent conservateur utilisé, et caisses pour le transport des échantillons non réfrigérés.

\section{Protocole expérimental}

Les différents paramètres étudiés pour la conservation ont été reportés au tableau I. Un total de 25 échantillons de lait individuel de $2500 \mathrm{ml}$ ont été divisés en 42 aliquotes de $50 \mathrm{ml}$ qui, à leur tour, ont été divisées en trois groupes selon la nature de l'agent conservateur utilisé : 14 sans conservateur (SC), 14 avec dichromate de potassium (DP) $(0,1 \mathrm{~g} / 100 \mathrm{ml})$ et 14 avec bronopol (BR) $(0,05 \mathrm{~g} / 100 \mathrm{ml})$. Deux aliquotes de chaque groupe ont été analysées sans stockage, à la température ambiante (TA), $5 \mathrm{~h}$ après collecte des échantillons. Les 12 aliquotes restantes ont été stockées à température réfrigérante (TR) $\left(4^{\circ} \mathrm{C}\right)$ et analysées 1,2 , $3,4,5$ et 6 jours après la première analyse des échantillons âgés de $5 \mathrm{~h}$. Ci-après, « jour d'analyse » fait référence au temps écoulé depuis la première analyse tout en sachant que pour connaître l'âge réel de l'échantillon il faut ajouter cinq heures. Le nombre d'aliquotes analysées par groupe et par âge a été donc de deux, l'une transportée sans conditionnement (TNC) et l'autre transportée dans une glacière avec accumulateur de froid (TC). Celle du premier groupe, âgée de $5 \mathrm{~h}$ et transportée sans conditionnement, c'est-à-dire SC, TA, TNC, a été utilisée comme référence. Tous les échantillons ont été analysés après chauffage dans un bain-marie à $40{ }^{\circ} \mathrm{C}(14,16)$.

L'expérience a été faite à deux reprises, l'une en début de lactation sur les 25 vaches (expérience 1) et l'autre deux mois après, en pleine lactation, sur 22 des mêmes vaches (expérience 2). Le nombre total d'observations a été respectivement de 1050 et 924 pour la première et la deuxième expérience.

\section{Analyse du lait}

Les SCC ont été déterminées par la méthode fluoro-optoélectronique à l'aide d'un appareil Fossomatic 5000, étalonné par rapport à la méthode de microscopie directe comme méthode de référence.

\section{Analyse des données}

L'analyse des données a été réalisée à deux niveaux. Dans un premier temps, les auteurs ont cherché à mettre en évidence le niveau de signification de chacun des facteurs considérés. Pour cela, les données ont été regroupées selon les différents niveaux des facteurs de variation contrôlés, jugés susceptibles d'affecter le comptage cellulaire du même lait individuel analysé à plusieurs reprises dans le temps, c'est-à-dire, aux différents âges de l'échantillon. Pour des raisons d'hypothèse statistique, les données de SCC ont subi une transformation logarithmique (LnSCC) $(1,16)$. Le traitement statistique des données a été effectué avec le logiciel SAS, vers. 6.1, à l'aide du modèle linéaire généralisé pour l'analyse de la variance et la comparaison des moyennes aux différents âges des échantillons. Chaque performance a été décomposée grâce à un modèle linéaire où l'effet vache est aléatoire et les autres sont fixés :

$\mathrm{Y}_{\mathrm{ijkl}}=\mu+\mathrm{V}_{\mathrm{i}}+\mathrm{T}_{\mathrm{j}}+\mathrm{C}_{\mathrm{k}}+\mathrm{TC}_{\mathrm{jk}}+\mathrm{e}_{\mathrm{ijkl}}$

\section{Tableau I}

Schéma descriptif du protocole expérimental à partir d'échantillons de laits individuels (42 aliquotes/vache)

\begin{tabular}{lcccc} 
Agent conservateur & Stockage & Age de l'échantillon & Réfrigération & Température d'analyse $\left({ }^{\circ} \mathrm{C}\right)$ \\
\hline 14 aliquotes sans conservateur & 2 à TA & \\
& 12 à $4{ }^{\circ} \mathrm{C}$ & $1,2,3,4,5,6 \mathrm{j}$ & Non (1 aliquote/âge) & 40 \\
14 aliquotes avec dichromate de potassium & 2 à TA & $5 \mathrm{~h}$ & Oui (1 aliquote/âge) & 40 \\
& 12 à $4{ }^{\circ} \mathrm{C}$ & $1,2,3,4,5,6 \mathrm{j}$ & Non (1 aliquote/âge) & \\
14 aliquotes avec bronopol & 2 à TA & $5 \mathrm{~h}$ & Oui (1 aliquote/âge) & 40
\end{tabular}

${ }^{*}$ Température ambiante 
où $Y_{i j k l}$ est la variable dépendante LnSCC, $\mu$ la moyenne, $V_{i}$ l'effet vache (25 ou 22 niveaux selon l'expérience), $\mathrm{T}_{\mathrm{j}}$ l'effet type de transport (deux niveaux : TNC et TC), $\mathrm{C}_{\mathrm{k}}$ l'effet agent conservateur (trois niveaux : SC, DP et $\mathrm{BR}$ ), $\mathrm{TC}_{\mathrm{jk}}$ l'interaction type de transport $\mathrm{x}$ agent conservateur, et $\mathrm{e}_{\mathrm{ijkl}}$ l'effet aléatoire résiduel.

Dans un second temps, les pertes de précision associées à l'estimation du taux cellulaire aux analyses successives du même lait (à différents âges de stockage) ont été étudiées suivant la démarche décrite dans des études antérieures (9), basée sur la notion de régression linéaire simple. Les résultats issus des analyses aux différentes âges $(\mathrm{X})$ ont été alors comparés à celui de référence $(\mathrm{Y})$ par régression linéaire en utilisant le modèle :

$\mathrm{Y}=\mathrm{a}+\mathrm{bX}+\mathrm{E}$

où a est l'ordonnée à l'origine, b la pente ou coefficient de régression, et $\mathrm{E}$ l'erreur aléatoire.

La perte de précision associée à chaque analyse est estimée comme $1-\mathrm{R}^{2}$ et exprimée en pourcentage. Cette analyse statistique a traité les données brutes de cellules somatiques, sans transformation logarithmique, pour une comparaison juste des résultats issus des différentes analyses. En effet, après transformation logarithmique et étant donné l'allure de la courbe de la fonction logarithme, la différence de points entre le résultat de référence et celui du comptage postérieur dépendrait du niveau cellulaire dans le lait. Une différence de 100 cellules/ml par exemple pour un lait de $100.10^{3}$ cellules/ml se traduirait, après une transformation en logarithme népérien, par une différence de 0,005 point alors que la même différence de cellules se traduirait par une valeur cinq fois inférieure, 0,001 point, pour un lait de $500.10^{3}$ cellules $/ \mathrm{ml}$.

\section{RESULTATS}

\section{Comptage cellulaire}

Les statistiques élémentaires (moyenne et écart-type) du comptage cellulaire à différents âges des laits individuels prélevés à deux reprises durant la lactation sont rapportées dans le tableau II. Les valeurs moyennes du niveau cellulaire des laits étudiés pour la première et la deuxième analyses $(5 \mathrm{~h})$ étaient respectivement de 11,07 et 11,03, en expression logarithmique népérienne, ce qui revient à une numération de 181 et $132.10^{3}$ cellules $/ \mathrm{ml}$. Ces valeurs ont été en deçà du seuil cellulaire de discrimination de l'état d'infection chez la vache laitière $(7,17)$.

Le taux cellulaire moyen s'est réduit de 27 p. 100 entre le premier et le deuxième prélèvement (tableau II). Ceci pouvait être dû à un effet de concentration (dilution des cellules somatiques dans un lait plus abondant), étant donné la corrélation négative entre la numération cellulaire et la quantité de lait produite déjà rapportée chez les espèces bovine $(12,15)$, ovine $(5)$ et caprine (18). La figure 1 montre davantage de détails sur l'évolution du taux

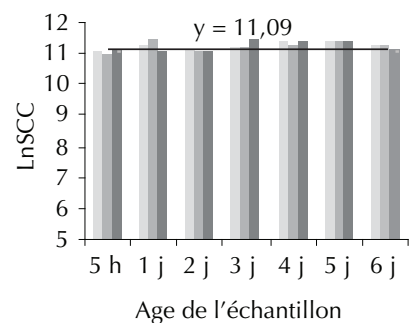

a. Début lactation, lait non réfrigéré

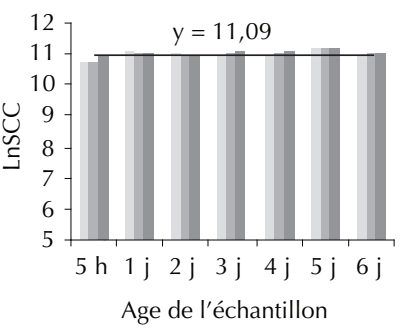

b. Début lactation, lait réfrigéré

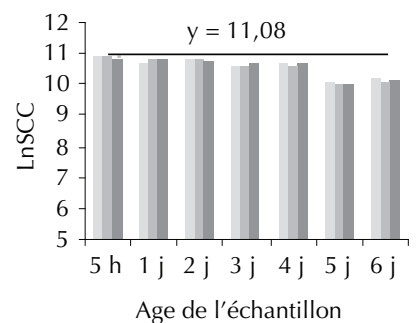

c. Pleine lactation, lait non réfrigéré

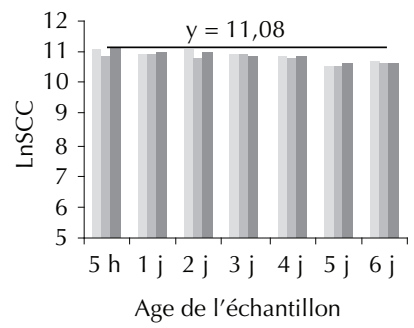

d. Pleine lactation, lait réfrigéré
Figure 1 : évolution du logarithme de la numération cellulaire (LnSCC) avec l'âge des échantillons pour les trois groupes de laits non conservés (gauche), avec dichromate (milieu), et bronopol (droite) ; "y » est la référence.

\section{Tableau II}

Moyennes arithmétiques et écarts-types (ET) du taux cellulaire dans des laits individuels à différents âges de stockage avant l'analyse des échantillons

\begin{tabular}{|c|c|c|c|c|c|c|c|c|}
\hline \multirow[t]{2}{*}{ Age (stockage) } & \multicolumn{4}{|c|}{ Début lactation (expérience 1) } & \multicolumn{4}{|c|}{ Pleine lactation (expérience 2) } \\
\hline & $\mathrm{n}$ & LnSCC & ET & SCC * & $\mathrm{n}$ & LnSCC & ET & SCC \\
\hline $5 \mathrm{~h}$ & 150 & 11,07 & 1,05 & 181 & 132 & 11,03 & 0,90 & 132 \\
\hline $1 \mathrm{j}$ & 150 & 11,30 & 1,04 & 210 & 132 & 10,95 & 0,81 & 107 \\
\hline $2 \mathrm{j}$ & 150 & 11,17 & 1,08 & 186 & 132 & 10,96 & 0,76 & 106 \\
\hline $3 j$ & 150 & 11,29 & 0,99 & 154 & 132 & 10,84 & 0,86 & 102 \\
\hline $4 j$ & 150 & 11,34 & 1,02 & 172 & 132 & 10,82 & 0,85 & 99 \\
\hline $5 \mathrm{j}$ & 150 & 11,42 & 1,19 & 284 & 132 & 10,37 & 1,04 & 85 \\
\hline $6 \mathrm{j}$ & 150 & 11,27 & 1,20 & 263 & 132 & 10,47 & 1,09 & 107 \\
\hline
\end{tabular}

* Comptage de cellules somatiques : moyenne arithmétique (x 103/ml) 
cellulaire avec l'âge des échantillons de lait en fonction du degré de réfrigération et de la nature de l'agent conservateur.

\section{Facteurs de variation}

Les résultats de l'analyse de la variance (tableau III) pour les facteurs de variation jugés susceptibles d'affecter le LnSCC de laits individuels analysés à différents moments indiquent que ces facteurs contrôlés ont expliqué un bon pourcentage de la variation du LnSCC, comme le montre la valeur élevée du coefficient de détermination $\left(\mathrm{R}^{2}\right)$ associée à chaque analyse, toujours supérieure à 80 p. 100.

La vache a été le seul facteur qui a contribué significativement à la variation des cellules somatiques pour les différentes analyses. Cependant, à quelques exceptions près, ni le type de transport ni la nature de l'agent conservateur ni leur interaction n'a eu d'effet sur l'évolution du LnSCC avec l'âge de l'échantillon. Lorsque le facteur type de transport montrait des effets significatifs (à 5 et 6 jours après prélèvement dans l'expérience 2), les valeurs moyennes des moindres carrés ont été plus élevées pour les laits à transport conditionné (respectivement 10,59 $\pm 0,041$ et 10,65 $\pm 0,044$, contre $10,15 \pm 0,041$ et 10,29 $\pm 0,044$ pour les laits âgés de 5 et 6 jours). Pour ce qui concernait la nature de l'agent conservateur, chaque fois que l'effet a été significatif $(\mathrm{P}<0,05)$, c'étaient les laits conservés avec BR qui avaient les LnSCC les plus élevés. Bien que ce phénomène ait été très rare dans la présente étude, il coïncidait avec les résultats de Gonzalo et coll. (9), et Martinez et coll. (14) sur le lait de brebis.

Les effets des facteurs de variations considérés ont été presque identiques d'une analyse à l'autre à cause peut-être de la faible variation du logarithme de la numération cellulaire au fil du temps, déjà rapportée ailleurs aussi bien chez les bovins (2) que chez les ovins (14) laitiers.

\section{Corrélation entre les mesures aux différentes analyses}

Les relations entre décomptes cellulaires au fil du temps durant les sept jours successifs (tableau IV) ont été étudiées en calculant les corrélations phénotypiques entre les mesures des différentes analyses en début de lactation (au-dessous de la diagonale) et en pleine lactation (au-dessus de la diagonale). Les corrélations ont été toutes élevées, allant de 0,49 à 0,89 pour la première expérience et de 0,64 à 0,86 pour la deuxième ; elles ont été plus élevées entre les mesures obtenues en pleine lactation, ce qui pouvait être lié au faible décompte cellulaire associé à une production laitière plus abondante.

Comme l'on pouvait s'y attendre, les valeurs des coefficients de corrélation les plus proches de la première diagonale ont été généralement les plus élevées. Cela signifiait que les résultats issus de deux analyses successives ont été plus corrélés que ceux relatifs à d'autres analyses plus échelonnées dans le temps.

\section{Précision des estimations}

Le tableau $\mathrm{V}$ rapporte les pertes de précision résultant de la comparaison des différentes analyses de cellules somatiques à celle du lait âgé de $5 \mathrm{~h}$, non réfrigéré et non conservé, prise comme référence.

Les résultats obtenus ont été différents selon le stade de lactation, le taux cellulaire étant négativement corrélé à la quantité de lait

\section{Tableau III}

Effets des facteurs de variation étudiés sur le comptage cellulaire (LnSCC) aux différents âges des échantillons de lait en début et en pleine lactation

\section{Source de variation}

$$
\text { ddI }
$$

\section{Début de lactation (expérience 1)}

\begin{tabular}{|c|c|c|c|c|c|c|c|c|}
\hline & & $5 \mathrm{~h}$ & $1 \mathrm{j}$ & $2 \mathrm{j}$ & $3 \mathbf{j}$ & $4 \mathrm{j}$ & $5 \mathbf{j}$ & $6 \mathrm{j}$ \\
\hline Individu & 24 & $17,97^{* * *}$ & $23,70^{* * *}$ & $26,79^{* * *}$ & $48,87^{* * *}$ & $45,55^{* * *}$ & $63,09^{* * *}$ & $56,41^{* * *}$ \\
\hline Transport & 1 & $0,28^{N S}$ & $0,27^{N S}$ & $2,22 \mathrm{NS}$ & $0,53 \mathrm{NS}$ & $2,58 \mathrm{NS}$ & $1,85^{N S}$ & 0,02 NS \\
\hline Conservation & 2 & $1,56 \mathrm{NS}$ & 2,02 NS & $0,13^{N S}$ & $4,70^{*}$ & $0,87^{N S}$ & 0,02 NS & $0,32 \mathrm{NS}$ \\
\hline Transport x conservation & 2 & $0,13^{N S}$ & $1,73^{N S}$ & 0,03 NS & $0,79^{\mathrm{NS}}$ & $1,28^{\mathrm{NS}}$ & 0,21 NS & $1,11^{\mathrm{NS}}$ \\
\hline $\mathrm{R}^{2}(\%)$ & & 78 & 83 & 84 & 91 & 90 & 93 & 92 \\
\hline & \multicolumn{8}{|c|}{ Pleine lactation (expérience 2) } \\
\hline Individu & 21 & $39,19^{* * *}$ & $35,63^{* * *}$ & $40,48^{* * *}$ & $26,10^{* * *}$ & $81,78^{* * *}$ & $54,38^{* * *}$ & $51,43^{* * *}$ \\
\hline Transport & 1 & $0^{\mathrm{NS}}$ & $0,21 \mathrm{NS}$ & $0,27^{N S}$ & $2,52 \mathrm{NS}$ & 1,09 NS & $57,87^{* * *}$ & $33,72^{* * *}$ \\
\hline Conservation & 2 & $2,07^{\mathrm{NS}}$ & $2,11^{\mathrm{NS}}$ & $3,52^{*}$ & 0,08 NS & $2,77^{\mathrm{NS}}$ & 0,06 NS & $0,48^{\mathrm{NS}}$ \\
\hline Transport x conservation & 2 & $5,24^{* *}$ & 0,39 NS & $2,41 \mathrm{NS}$ & $0,12^{N S}$ & $0,2 \mathrm{NS}$ & $0,23 \mathrm{NS}$ & $0,27^{\mathrm{NS}}$ \\
\hline $\mathrm{R}^{2}(\%)$ & & 89 & 88 & 89 & 84 & 94 & 92 & 91 \\
\hline
\end{tabular}


Table IV

Coefficients de corrélation entre cellules somatiques à différents âges des échantillons de laits individuels prélevés en début (au-dessous de la diagonale) et en pleine lactation (au-dessus de la diagonale) de vaches Holstein

\begin{tabular}{|c|c|c|c|c|c|c|c|}
\hline Variable $(\mathbf{i} / \mathbf{j})^{*}$ & 1 & 2 & 3 & 4 & 5 & 6 & 7 \\
\hline $1 \mathrm{LnSCC}(5 \mathrm{~h})$ & & 0,83 & 0,81 & 0,77 & 0,81 & 0,80 & 0,74 \\
\hline $2 \operatorname{LnSCC}(1 \mathrm{j})$ & 0,67 & & 0,83 & 0,77 & 0,82 & 0,78 & 0,80 \\
\hline $3 \operatorname{LnSCC}(2 \mathrm{j})$ & 0,64 & 0,61 & & 0,82 & 0,84 & 0,77 & 0,76 \\
\hline $4 \operatorname{LnSCC}(3 \mathrm{j})$ & 0,49 & 0,38 & 0,59 & & 0,84 & 0,69 & 0,64 \\
\hline $5 \operatorname{LnSCC}(4 \mathrm{j})$ & 0,52 & 0,38 & 0,61 & 0,86 & & 0,78 & 0,76 \\
\hline $6 \operatorname{LnSCC}(5 \mathrm{j})$ & 0,55 & 0,49 & 0,66 & 0,86 & 0,89 & & 0,86 \\
\hline $7 \operatorname{LnSCC}(6 \mathrm{j})$ & 0,58 & 0,51 & 0,67 & 0,79 & 0,81 & 0,89 & \\
\hline
\end{tabular}

${ }^{*} \mathrm{i} / \mathrm{j}=$ variable $\mathrm{i}$ et variable $\mathrm{j}$

\section{Table V}

Pertes de précision $\left(1-\mathrm{R}^{2}\right)$ et coefficients de régression (b) pour les différentes analyses par rapport à celle de référence (fond gris foncé : sans réfrigération, sans conservateur, sans stockage)

\begin{tabular}{|c|c|c|c|c|c|c|c|c|c|c|c|c|c|}
\hline \multirow[b]{3}{*}{ Critère } & \multirow[b]{3}{*}{ Age } & \multicolumn{6}{|c|}{ Début de lactation } & \multicolumn{6}{|c|}{ Pleine lactation } \\
\hline & & \multicolumn{3}{|c|}{ Non réfrigéré } & \multicolumn{3}{|c|}{ Réfrigéré } & \multicolumn{3}{|c|}{ Non réfrigéré } & \multicolumn{3}{|c|}{ Réfrigéré } \\
\hline & & SC & DP & BR & SC & DP & BR & SC & DP & BR & SC & DP & BR \\
\hline \multirow{7}{*}{$1-R^{2}$} & $5 \mathrm{~h}$ & 0 & 0 & 24 & 6 & 8,3 & 6,1 & 0 & 0,5 & 0,4 & 4 & 3,4 & 1,3 \\
\hline & $\mathrm{J} 1$ & 3,84 & 43,1 & 6,7 & 18,7 & 19,3 & 47 & 3,8 & 4,7 & 4,1 & 1,6 & 2 & 2,3 \\
\hline & $\mathrm{J} 2$ & 15 & 11,2 & 21,2 & 9,3 & 9,8 & 16,1 & 3,7 & 5,3 & 4,5 & 5,6 & 2 & 4,6 \\
\hline & $\mathrm{J} 3$ & 18 & 20,1 & 14,3 & 17 & 15,1 & 25,3 & 3,8 & 4,1 & 4,1 & 5,4 & 4,3 & 4,6 \\
\hline & $\mathrm{J} 4$ & 18,4 & 19,8 & 54,3 & 19,3 & 19 & 23,1 & 4,1 & 3,5 & 3,6 & 3,6 & 2,8 & 5,1 \\
\hline & $\mathrm{J} 5$ & 77,4 & 66,8 & 78,4 & 11,8 & 26,7 & 79,7 & 3,8 & 3,5 & 3,6 & 4,7 & 3,9 & 3,9 \\
\hline & J6 & 50,6 & 85,8 & 79,2 & 19,7 & 93,3 & 71,1 & 5,1 & 5,6 & 6,1 & 5,2 & 4,2 & 5,7 \\
\hline \multirow{7}{*}{ b } & $5 \mathrm{~h}$ & 1 & 0,948 & 0,876 & 0,584 & 0,598 & 1,024 & 1 & 1,011 & 0,860 & 0,505 & 0,575 & 0,849 \\
\hline & $\mathrm{J} 1$ & 0,564 & 0,941 & 0,616 & 0,914 & 0,979 & 0,531 & 1,013 & 0,995 & 1,003 & 0,974 & 1,026 & 0,862 \\
\hline & $\mathrm{J} 2$ & 0,945 & 0,879 & 1,412 & 0,543 & 0,670 & 1,032 & 1,008 & 0,902 & 0,972 & 0,994 & 1,009 & 0,932 \\
\hline & $\mathrm{J} 3$ & 1,505 & 1,564 & 1,018 & 1,433 & 1,389 & 1,450 & 1,001 & 0,797 & 1,006 & 0,997 & 0,999 & 0,993 \\
\hline & $\mathrm{J} 4$ & 1,445 & 1,430 & 0,494 & 1,438 & 1,378 & 1,350 & 1,002 & 0,965 & 1,015 & 0,994 & 1,005 & 0,983 \\
\hline & $\mathrm{J} 5$ & 0,399 & 0,443 & 0,252 & 0,770 & 0,408 & 0,221 & 1,005 & 1,005 & 1,003 & 1,002 & 1,002 & 1,014 \\
\hline & $J 6$ & 0,239 & 0,238 & 0,272 & 1,454 & 0,201 & 0,585 & 0,669 & 0,709 & 0,783 & 0,770 & 0,675 & 0,839 \\
\hline
\end{tabular}

SC : sans conservateur ; DP : dichromate de potassium ; BR : bronopol

produite et très variable dans le temps $(1,6,15,19)$. Une bonne précision des estimations a été souvent observée (> 95 p. 100) pour les laits prélevés en pleine lactation où le taux cellulaire est moins élevé (moyenne de $132.10^{3}$ cellules $/ \mathrm{ml}$ ), et ce, pour les sept temps d'analyse et indépendamment de la réfrigération et de la conservation du lait. Il n'en a pas été de même pour les laits prélevés en début de lactation avec des taux cellulaires plus élevés $\left(181.10^{3}\right.$ cellules/ml). En effet, si à quelques exceptions près, les pertes de précision ont été acceptables (de l'ordre de 10 p. 100) durant les premières 48 heures, elles sont devenues plus importantes au troisième jour et excessivement élevées pour les trois jours suivants.

En général, il n'a pas été observé de tendance claire d'amélioration de la précision associée à la réfrigération des laits durant leur transport de la ferme au laboratoire d'analyse. De même, la nature de l'agent conservateur n'a pas eu d'effet sur la précision des estimations, aussi bien en début qu'en pleine lactation, même si une tendance à de meilleurs résultats a été parfois observée avec le dichromate de potassium par rapport au bronopol, surtout pour les laits réfrigérés durant le transport. 


\section{DISCUSSION}

La relation entre les cellules somatiques et la qualité physicochimique du lait est un fait bien connu, compte tenu de la variabilité de son aptitude à la transformation induite par les modifications du taux cellulaire à la production, avec le stade de lactation ou en fonction d'autres facteurs infectieux ou non. Cependant, bien d'autres facteurs fins liés aux circonstances spécifiques à chaque contrôle laitier peuvent affecter le niveau cellulaire entre la production et l'analyse du lait. L'un des objectifs de ce travail a été de déterminer les délais seuils après prélèvement pendant lesquels le lait pourrait être analysé sans que sa qualité cellulaire ne soit trop altérée.

Le faible niveau de cellules somatiques dans le lait est probablement dû à l'effort fourni au sein de l'élevage (antibiothérapie de tarissement, trempage des trayons, vulgarisation, etc.) ces dernières années pour l'amélioration des conditions de production. L'information disponible ne permet pas de vérifier si l'amélioration de la qualité a été régulière pendant ces dernières années : l'amélioration sensible expérimentée a été plus marquée à partir des années 1990, date de l'inclusion de l'analyse en routine de la numération cellulaire dans le contrôle laitier officiel. Quoi qu'il en soit, le comptage de cellules somatiques du lait produit par le troupeau étudié serait en moyenne inférieur à celui du lait collecté dans la zone.

Le dispositif expérimental appliqué dans cette étude a permis de constater que parmi les facteurs de variation envisagés, seul le facteur individu a eu un effet très significatif sur le LnSCC dans les laits individuels. L'absence d'effet de l'agent conservateur ou des conditions de transport était peu surprenante du fait qu'il s'agissait, d'une part, de mesures répétées des mêmes échantillons de lait et, d'autre part, de mesures transformées en logarithme pour des hypothèses statistiques. En d'autres termes, ni la réfrigération ni la conservation du lait ne semblaient maintenir mieux sa qualité cellulaire initiale par rapport à celui non réfrigéré non conservé.

A priori et à en juger par les résultats de cette étude, les pertes de précision associées au temps écoulé depuis le prélèvement des échantillons par rapport à la valeur initiale des cellules somatiques ont été en relation évidente avec le niveau cellulaire dans le lait. Une autre indication sur cette relation était la différence entre coefficients de corrélation entre mesures associées à chaque expérience, plus élevées en pleine lactation. Autrement dit, les délais dans lesquels le décompte de cellules somatiques peut être mesuré après prélèvement du lait dépendraient du stade de lactation et de l'état de santé des mamelles dans l'élevage laitier concerné. La période de stockage seuil de 48 h observée durant la première expérience, où le taux cellulaire était plus élevé, rejoint les résultats de Gonzalo et coll. (8) sur le lait de brebis avec un taux cellulaire de l'ordre de $500.10^{3}$ cellules $/ \mathrm{ml}$. Ces derniers ont fixé des délais seuils respectivement de 12 h et 2 jours pour des aliquotes non réfrigérées ni conservées et des aliquotes stockées à $4{ }^{\circ} \mathrm{C}$ sans conservation. Pour des raisons pratiques, et même si toute la période de stockage étudiée reste valable en pleine production de la vache, il est recommandé de veiller à effectuer les analyses cellulaires dans les deux jours suivant le prélèvement des échantillons, et ce, indépendamment du stade de lactation.

Ces considérations permettent de penser que les résultats de cette étude ne seraient valables que pour les élevages laitiers à taux cellulaire aussi faible. En outre, les données publiées de façon éparse sur la question laissent comprendre que le problème de la mammite est difficile à cerner ; il s'agit d'une maladie causée par plusieurs facteurs $(4,11)$. Les microorganismes sont responsables de l'infection, mais pour que ceux-ci entrent dans les glandes mammaires et qu'ils s'établissent au point de provoquer une infection, de nombreux facteurs peuvent intervenir. Ces facteurs (hygiène, stabulation, climat, trayeuses, alimentation, génétique, etc.) agissent tous en même temps. Il est de plus difficile de généraliser quant à l'importance relative de chacun de ces facteurs, certains facteurs étant spécifiques à certains microorganismes. Klastrup et coll. (13) estiment que 25 p. 100 de la susceptibilité aux infections sont attribuables aux facteurs environnementaux, $20 \mathrm{p}$. 100 aux facteurs génétiques et 50 p. 100 à la conduite du troupeau. Ce qui prouve que l'interprétation de tels résultats dépendrait des modes de conduite au sein de chaque troupeau ou population.

\section{CONCLUSION}

Pour un même lait, des échantillons analysés à différents âges durant sept jours successifs, la réfrigération durant le transport et la nature de l'agent conservateur n'ont pas eu d'effets significatifs sur le LnSCC. La cohérence entre les différentes analyses était liée au stade de lactation, c'est-à-dire au niveau cellulaire dans le lait. Quand les SCC étaient faibles, de l'ordre de $132.10^{3}$ cellules $/ \mathrm{ml}$, les pertes de précision associées aux analyses aux différents âges des échantillons par rapport à celles âgée de $5 \mathrm{~h}$ étaient faibles et donc statistiquement acceptables. Pour la première expérience, où le niveau cellulaire dans le lait était plus élevé $\left(181.10^{3}\right.$ cellules/ $\mathrm{ml})$, les cellules somatiques mesurées après $48 \mathrm{~h}$ ont subi des déviations importantes par rapport à celle de référence. Pour des raisons pratiques et dans les conditions actuelles du troupeau sujet de l'étude, l'analyse des laits individuels pour les cellules somatiques au sein du contrôle laitier officiel devrait donc être effectuée au plus tard deux jours après le prélèvement des échantillons.

\section{Remerciements}

Cette étude a été financée par le ministère de la Recherche scientifique et de la Technologie (Tunis, Tunisie). Nous tenons aussi à remercier l'équipe de la SFL pour sa collaboration qui a permis de réaliser la traite et le prélèvement des échantillons selon les conditions exigées.

\section{BIBLIOGRAPHIE}

1. ALI A.K., SHOOK G.E., 1980. An optimum transformation for somatic cell concentration in milk. J. Dairy Sci., 63: 487-490.

2. BARKEMA H.W., VAN DER SCHANS J., SCHUKKEN Y.H., DE GEE A.L.W., LAM T.J.G.M., BENEDICTUS G., 1997. Effect of freezing on somatic cell count of quarter milk samples as determined by a Fossomatic electronic cell counter. J. Dairy Sci., 80: 422-426.

3. CARAVIELLO D.Z., WEIGEL K.A., SHOOK G.E., RUEGG P.L., 2005. Assessment of the impact of somatic cell count on functional longevity in Holstein and Jersey cattle using survival analysis methodology. J. Dairy Sci., 88: 804-811.

4. DUVAL J., 1995. Soigner la mammite sans antibiotiques. Ste-Anne-deBellevue, Canada, Ecological Agriculture Projects, p. 370-411.

5. EL-SAIED U.M., CARRIEDO J.A., DE LA FUENTE L.F., SAN PRIMITIVO F., 1999. Genetic parameters of lactation cell counts and milk and protein yields in dairy ewes. J. Dairy Sci., 82: 639-644.

6. GAMBO H., AGNEM ETCHIKE C., 2001. Dépistage de mammites subcliniques chez des vaches Goudali en lactation au Nord Cameroun. Revue Elev. Méd. vét. Pays trop., 54 : 5-10

7. GONZALO C., GONZALEZ M.C., ARIZNABARRETA.A., SAN PRIMITIVO F., 1998. Umbral celular de discriminación de la infección mamaria ovina. Ovis, 56: 41-46.

8. GONZALO C., MARTINEZ J.R., CARRIEDO J.A., SAN PRIMITIVO F., 2003. Fossomatic cell counting on ewe milk: comparison with direct microscopy and study of variation factors. J. Dairy Sci., 86: 138-145. 
9. GONZALO C., OTHMANE M.H., FUERTES J.A., DE LA FUENTE L.F., SAN PRIMITIVO F., 2003. Losses of precision associated with simplified designs of milk recording for dairy ewes. J. Dairy Res., 70: 441-444.

10. GOTTSCHALK M., 2000. Nouveaux outils de laboratoire pour le diagnostic de la mammite. In : $2^{\mathrm{e}}$ rencontre de la voie lactée, conf. de Lennoxville sur la production laitière "la science au service de I'industrie », Sherbrooke, Québec, jan. 2000.

11. KENNEDY B.W., SETHAR M., TONGA A., MOXLEY J., 1982. Environmental factors influencing test day somatic cell counts in Holsteins. J. Dairy Sci., 65: 275-280.

12. KIRK J.H., 1984. Programmable calculator program for linear somatic cell counts scores to estimate mastitis field losses. J. Dairy Sci., 67: 441444 .

13. KLASTRUP O., BAKKEN G., BRAMLEY J., BUSHNELL R., 1987. Environmental influences on bovine mastitis. Bull. int. dairy Fed. (217), $37 \mathrm{p}$.

14. MARTINEZ J.R., GONZALO C., CARRIEDO J.A., SAN PRIMITIVO F., 2003. Effect of freezing on Fossomatic cell counting in ewe milk. J. Dairy Sci., 86: 2583-2587.

\section{Summary}

Othmane M.H., Trabelsi L., Ben Hammouda M., Bergaoui R. Effect of Preservation and Transport Conditions on SCC in Cow Milk and Losses of Precision Associated with Time Left Before Analysis

The effect of preservation agents (without preservation, potassium dichromate, and bronopol) on the somatic cell count (SCC) of individual cow milk samples was studied at two different times during lactation. Using the fossomatic method, 1050 (experiment 1) and 924 (experiment 2) SCC measurements were carried out on milk samples from 25 and 22 cows of the same lot, respectively. In addition, each individual milk sample was divided into several aliquots and analyzed at different times postcollection (5h, and 1, 2, 3, 4, 5 and 6 days) to evaluate the loss of precision associated to each milk age, depending on whether aliquots were preserved, refrigerated, or not refrigerated. Preservation and refrigeration during milk transport had no significant effects on SCCs in both experiments, which differed in milk SCC levels. Accuracy associated to milk age depended on the lactation stage; errors were much lower when milk was more abundant and the SCC level was thus lower. For practical reasons, milk analysis for SCC should be carried out at the latest $48 \mathrm{~h}$ postcollection.

Keywords: Cattle - Cow milk - Cell count - Refrigeration Preservation - Tunisia.
15. NG-KWAI-HANG K.F., HAYES J.F., MOXLEY J.E., MONARDES H.G., 1984. Variability of test-day milk production and composition and relation of somatic cell counts with yield and compositional changes of bovine milk. J. Dairy Sci., 67: 361-366.

16. OTHMANE M.H., CARRIEDO J.A., SAN PRIMITIVO F., DE LA FUENTE L.F., 2002. Genetic parameters for lactation traits of milking ewes: protein content and composition, fat, somatic cells and individual laboratory cheese yield. Genet. Sel. Evol., 34: 581-596.

17. SCHULTZ L.H., 1977. Somatic cells in milk: physiological aspects and relationships to amount and composition of milk. J. Food Prot., 40: 125-131.

18. UPADHYAYA T.N., RAO A.T., 1993. Diagnosis and threshold values of subclinical mastitis in goats. Small Ruminant Res., 12: 201-210.

19. VICARIO D., DEGANO L., CARNIER P., 2005. Test-day model for national genetic evaluation of somatic cell count in Italian Simmental population. Interbull, 33: 171-180.

Reçu le 04.04.2007, accepté le 08.02.2008

\section{Resumen}

Othmane M.H., Trabelsi L., Ben Hammouda M., Bergaoui R. Efecto de las condiciones de transporte y de la conservación sobre el conteo celular y las pérdidas de precisión asociadas con las edades de las muestras de leche de vaca

Se estudio el efecto del agente conservador (sin conservación, dicromato de potasio o bronopol) y de las condiciones de transporte (temperatura ambiente y refrigeración) sobre las tasas celulares (SCC) de las leches individuales de vacas, en dos ocasiones durante la lactación. Se efectuaron 1050 (experimento 1) y 924 (experimento 2) medidas de SCC gracias a una máquina Fossomatic, a partir de leches individuales, respectivamente de 25 y de 22 vacas de un mismo lote. Además, cada leche individual se dividió en varios alícuotas y se analizó en diferentes momentos después de la toma de la muestra ( $5 \mathrm{~h}$ y $1,2,3,4,5$ y 6 días post muestreo) para estimar las pérdidas de precisión asociadas con el tiempo de análisis, según si los alícuotas son conservados, refrigerados o no. $\mathrm{Ni}$ el agente conservador ni las condiciones de transporte tuvieron efectos significativos sobre la tasa celular en ninguna de las dos experiencias, que difirieron por el nivel celular en la leche. La precisión de las medidas, asociada a cada edad, dependía del estadio de lactación, los errores fueron mucho más bajos cuando la cantidad de la leche era más abundante y la SCC era así menos elevada. Por razones prácticas, el análisis de la leche para la SCC, debería ser efectuado a más tardar 48 h después de tomada la muestra.

Palabras clave: Ganado bovino - Leche de vaca - Conteo de células - Refrigeración - Preservación - Túnez. 